\title{
Direct Transformation from Arylamines to Aryl Naphthalene-1,8-diamino Boronamides: A Metal-Free Sandmeyer-Type Process
}

\author{
Siyi Ding $\mathbb{D}^{\mathbb{D}}$, Qiang Ma, Min Zhu, Huaping Ren, Shaopeng Tian, Yuzhen Zhao and \\ Zongcheng Miao *
}

Key Laboratory of Organic Polymer Photoelectric Materials, School of Science, Xijing University, Xi'an 710123, China; dingsiyi2009@163.com (S.D.); coobi@163.com (Q.M.); zhum_588@163.com (M.Z.); hpren2034@163.com (H.R.); tspspirit@163.com (S.T.); zyz19870226@163.com (Y.Z.)

* Correspondence: miaozongcheng@xijing.edu.cn; Tel.: +86-189-9115-0632

Received: 21 December 2018; Accepted: 16 January 2019; Published: 22 January 2019

check for updates

\begin{abstract}
A direct metal-free transformation from arylamines to aryl naphthalene-1,8-diamino boronamides, a type of masked boronic acid, has been developed based on Sandmeyer-type reactions. A nonsymmetrical diboron reagent, $\mathrm{B}$ (pin)-B(dan), was utilized as the borylating reagent, and the $\mathrm{B}$ (dan) moiety was transferred to the aim products selectively. This conversion tolerated a series of functional groups, including chloro, bromo, fluoro, ester, hydroxy, cyano and amide.
\end{abstract}

Keywords: boronamides; sandmeyer-type reaction; metal-free borylation

\section{Introduction}

Organoboron compounds, because of their increasing utilization in synthetic chemistry, drug discovery and materials science, have attracted significant attention in recent years [1]. They can react with various functional groups to construct new carbon-carbon bonds or carbon-heteroatom bonds, which can rapidly construct the complex structures of target molecules [2,3]. Therefore, much effort has been devoted to the exploration of synthesizing organoboron compounds [4-6]. On the other hand, to take best advantage of their diverse reactivity, chemists have also focused on adjusting their reactivity by varying masking groups on the boron atoms [2]. When naphthalene-1,8-diaminato (dan) ligand is used as the masking group, which has two nitrogen atoms that may donate their lone pair electrons to the vacant $p$-orbital of the boron atoms, the formed naphthalene-1,8-diamino boronamide (Bdan) compounds are robust enough to avoid undesirable organic reactions, such as Suzuki-Miyaura coupling reactions. Moreover, such compounds can be easily transformed to their corresponding boronic acids by simple treatment under aqueous acidic conditions. These features enable their wide application as modular synthetical building blocks $[4,7,8]$, especially in the iterative cross-coupling reactions [9-12] and the application of di-boron compounds [13-18].

When it comes to the synthesis of aryl B(dan) compounds, the most common strategy is condensation between commercially available aryl boronic acids and 1,8-diaminonaphthalene in refluxing toluene (Scheme 1, Path 1) [9]. If aryl halides are used as the starting materials, free boronic acids can be initially obtained via the traditional reactions between aryl magnesium, or -lithium, which derive from metal halogen exchanges, with trimethyl borate followed by hydrolytic workup (Scheme 1, Path 2). Then, aryl B(dan) compounds are accessible via the condensation process (Scheme 1, Path 1). The common products of Miyaura borylation reactions of aryl halides via the catalysis of transition metal such as Pd [19,20], Cu [21] and Ni [22,23] (Scheme 1, Path 3), aryl B(pin), can be converted to their $\mathrm{B}(\mathrm{dan})$ derivatives in the presence of $\mathrm{FeCl}_{2}$ [24] (Scheme 1, Path 4). In addition 
to these two indirect approaches, in 2015, $\mathrm{Xu}$ and Li reported a direct synthetical pathway of aryl $\mathrm{B}$ (dan) from aryl halides [25], which utilized a Pd-catalyzed selective boronyl transfer process of the non-symmetrical diboron reagent B(pin)-B(dan) (Scheme 1, Path 5). Later, similarly processed catalyzed $\mathrm{Cu}$ was also proved to be feasible by the Yoshida group [26,27]. As mentioned above, to the best of our knowledge, the synthesis of aryl B(dan) is largely dependent on the accessibility of aryl halides. Moreover, these approaches suffer from sensitivity to air and moisture, in view of the intermediation of aryl metallic compounds [28]. Such a situation inspired our exploration towards metal-free synthesis of aryl B(dan) compounds.

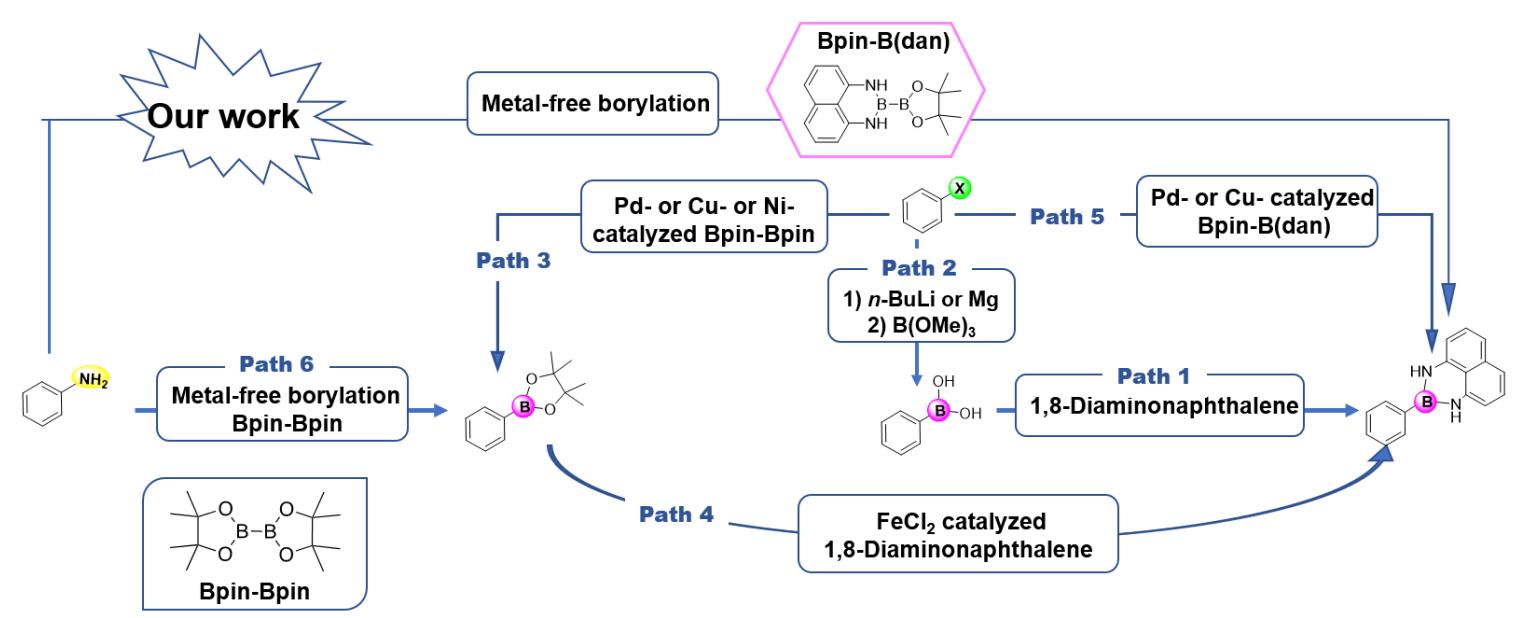

Scheme 1. Different routes to synthesize aryl B(dan) derivatives.

Arylamines, as cheap and abundant organic feedstock, can go through Sandmeyer-type reactions [29-32], being easily converted into various functional groups. Generally, two steps are required to complete the transformation from arylamines to aryl boronic acids or their derivatives. The amino groups can first be replaced by halides which are then utilized in the borylation reactions, as indicated in Scheme 1. Recently, Wang and co-workers reported a novel metal-free borylation method using arylamines as the starting materials [33-36] (Scheme 1, Path 6). In this process, symmetrical $\mathrm{B}$ (pin)-B(pin) was utilized as the borylating reagent [37-39]. In order to obtain aryl $\mathrm{B}$ (dan) from arylamines, an additional step is required to complete the conversion (Scheme 1, Path 4). So far, no one has ever realized the conversion from arylamines into their aryl B(dan) derivatives. It was envisaged that the nonsymmetrical $\mathrm{B}$ (pin)-B(dan) might be useful to achieve such a process [40-46]. Inspired by this idea, we sought to apply B(pin)-B(dan) in Sandmeyer-type reactions of arylamines.

\section{Results}

At the outset, we carried out the reactions under the reaction conditions that were similar to the previously reported Sandmeyer-type borylation reactions [47-62], with MeCN as the solvent. We chose 4-methoxyl aniline as the starting material, and no target product could be observed under the classical reaction conditions $[t-\mathrm{BuONO}, \mathrm{MeCN}$, room temperature]. However, by increasing the reaction temperature to $80{ }^{\circ} \mathrm{C}$, a low yield of $28 \%$ was obtained. As the Sandmeyer reaction was well known to involve a radical mechanism, we added the radical initiator benzoyl peroxide (BPO) in subsequent experiments. Consequently, 13\% of compound 1a could be obtained at room temperature, compared with $31 \%$ at $80{ }^{\circ} \mathrm{C}$ (Table 1 , entries 1-4). In view that a base could accelerate the trans-borylation of $\mathrm{B}_{2}$ pin 2 , we expected that a base could also play the same role for $\mathrm{B}$ (pin)-B(dan). Then, our expectations were met. After extensive variation of bases, the yield of reaction was significantly improved by the addition of NaOAc (Table 1, entries 5 and 18-20). Further experiments were conducted to improve the yield and it was noted that the addition of phase-transfer catalyst tetrabutylammonium iodide (TBAI) could enhance the yield of $\mathbf{1 a}$ to $75 \%$ (Table 1, entry 9). Lowering the loading of aryl amines was detrimental to this transformation, and 2.0 equivalent of 4-methoxyl aniline worked best in terms 
of reactivity (Table 1, entries 9, and 14-17). Finally, further assessment of the reaction temperature effect indicated that decreasing or increasing the temperature led to dramatically lower yields (Table 1 , entries 9-13).

Table 1. Optimization of reaction conditions.

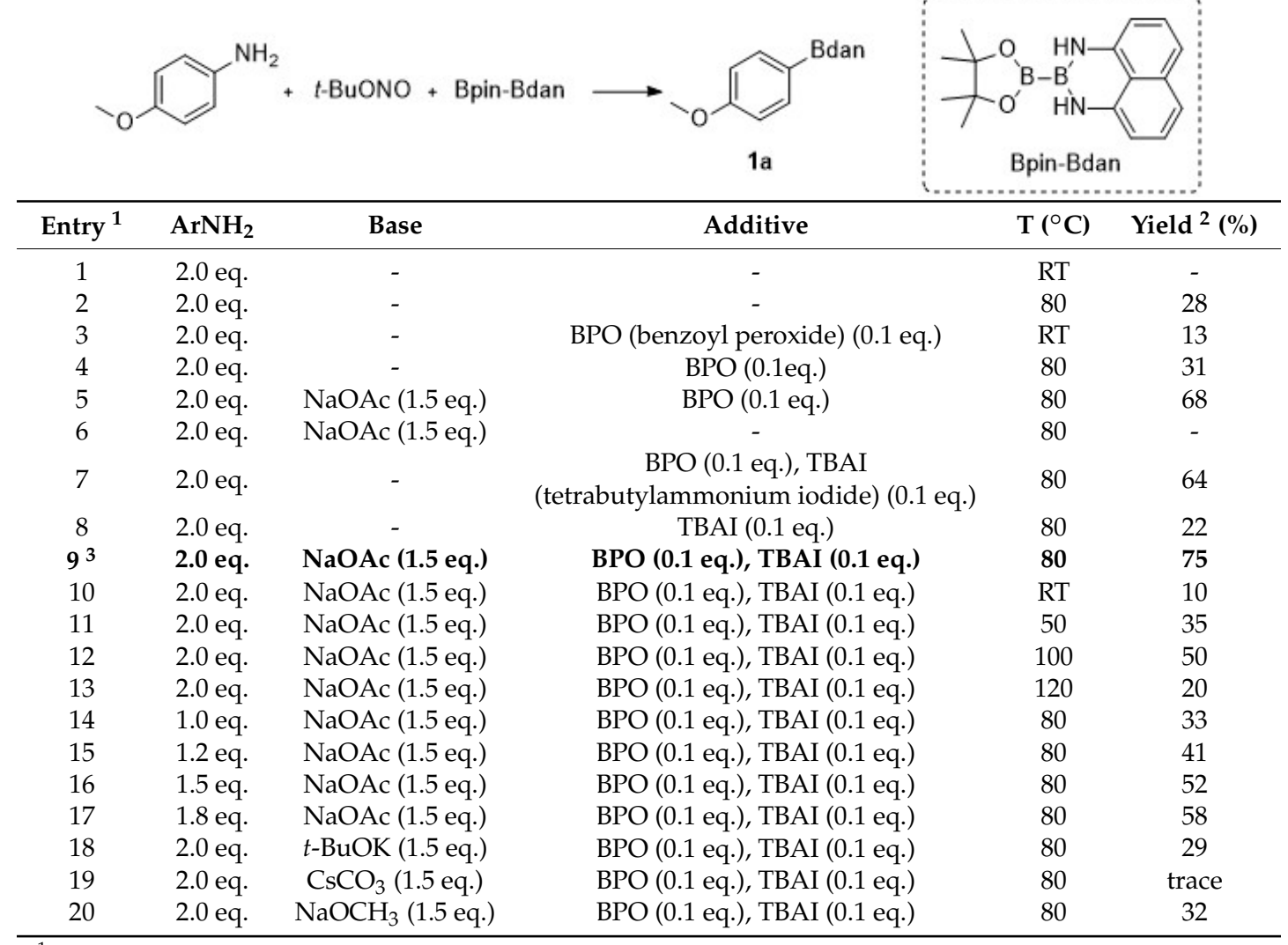

\footnotetext{
${ }^{1}$ Reaction conditions: aniline $(0.2 \mathrm{mmol}), t$-BuONO $(0.2 \mathrm{mmol})$, Bpin-B(dan) $(0.1 \mathrm{mmol}), \mathrm{MeCN}(0.6 \mathrm{~mL})$. The reaction was stirred in $\mathrm{N}_{2}$ atmosphere for $6 \mathrm{~h} .{ }^{2}$ Isolated yield. ${ }^{3}$ Entries in bold represent optimized reaction conditions.
}

Having defined an appropriate set of reaction conditions, we briefly investigated the scope of arylamines with various functional groups (Scheme 2). For most of the para-substituted arylamines (compounds 1a-1f) and meta-substituted substrates (compounds $\mathbf{1 g}-\mathbf{1 q}$ ), moderate yields were obtained. Then, relatively low yields of compounds $1 \mathrm{r}$ or $1 \mathrm{~s}$ were obtained, in which ortho-methylphenylamine and ortho-(ethoxycarbonyl)aniline were used. These results hinted that this reaction was sensitive to the steric hindrance. Compared with electron-rich arylamines, the anilines with electron-withdrawing groups showed higher reactivity. This new strategy featured broad functional-group tolerance. The anilines bearing functional groups, such as ester (compounds 1f, 11 and 1s), were suitable substrates. Additionally, the moieties with hydroxy (compound 2d), cyano (compound 1e) and amide (compound 1m) were compatible. Halogen atoms of arylamines, such as bromo (compound $\mathbf{1 b}, \mathbf{1} \mathbf{j}$ ), chloro (compound $\mathbf{1 q}$ ) and fluoro (compound $\mathbf{1 c}, \mathbf{1} \mathbf{i}, \mathbf{1} \mathbf{k}$ and $\mathbf{1} \mathbf{p}$ ) atoms, remained intact under the standard conditions, demonstrating the mild nature of the reaction condition. In order to confirm the practicality and potential application of this metal-free borylation process, the reaction was carried out on a $2.0 \mathrm{mmol}$ scale under the standard reaction conditions for selected substrates (ortho-, meta- and para-substituted compounds). The reactions provided the desired derivatives in moderate yields. 
(a)
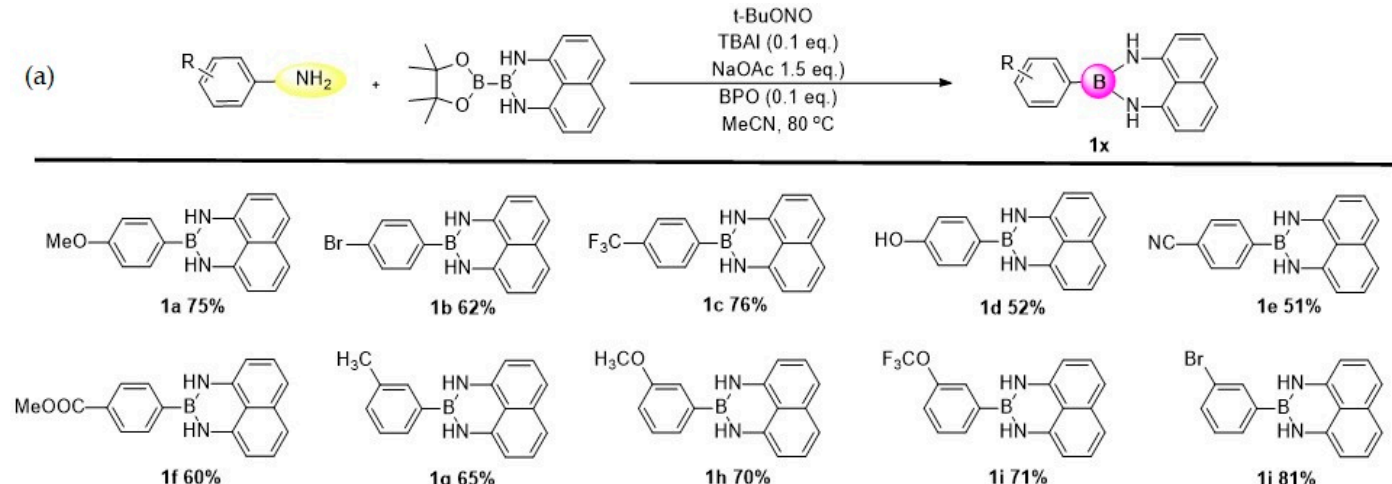

(b)

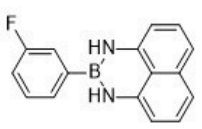

$\mathrm{MeOOC}$
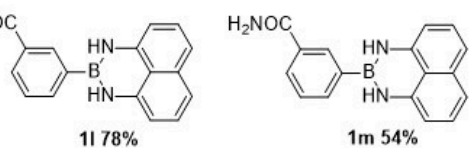

$1 \mathrm{~m} 54 \%$

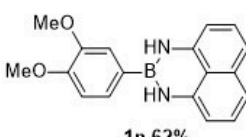

$1 \mathrm{j} 81 \%$
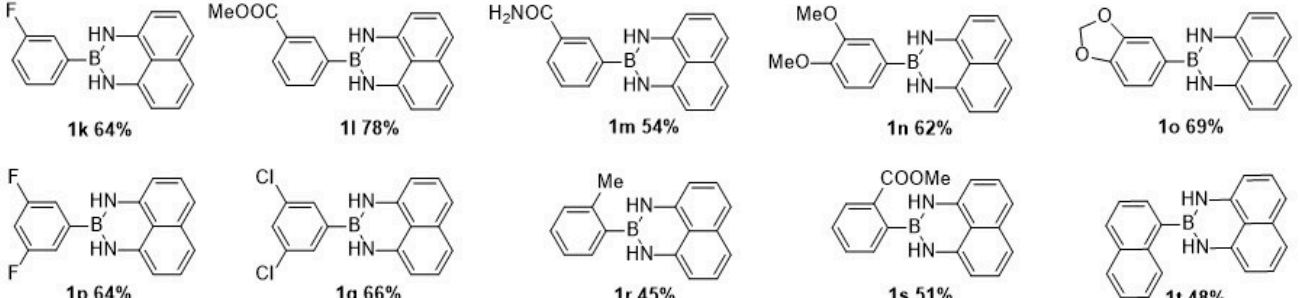

1o $69 \%$

1p $64 \%$

1q $66 \%$

1r $45 \%$

1s $51 \%$

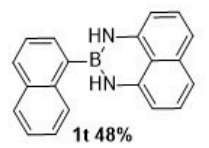

Large scale experiments

(c)

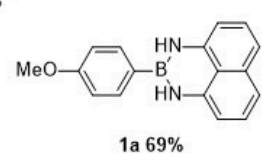

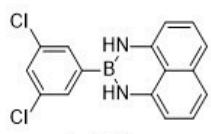

1q $60 \%$

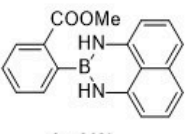

$1 \mathrm{~s} 41 \%$

Scheme 2. Different routes to synthesize aryl B(dan) derivatives. (a) Unless otherwise noted, the reaction conditions are as follows: aryl amine ( $0.2 \mathrm{mmol}, 2.0$ eq.), $\mathrm{B}$ (pin)-B(dan) (0.1 mmol, 1.0 eq.), $t$-BuONO (0.2 mmol, 2.0 eq.), TBAI (0.01 mmol, 0.1 eq.), NaOAc ( $0.15 \mathrm{mmol}, 1.5$ eq.), BPO (0.01 mmol, 0.1 eq.), $\mathrm{MeCN}(0.6 \mathrm{~mL}), 80^{\circ} \mathrm{C}$. (b) Yield of isolated product. (c) Large scale experiments, reaction conditions: aryl amine ( $2 \mathrm{mmol}, 2.0$ eq.), B(pin)-B(dan) (1 mmol, 1.0 eq.), $t$-BuONO ( 2 mmol, 2.0 eq.), TBAI (0.1 mmol, 0.1 eq.), NaOAc (1.5 mmol, 1.5 eq.), BPO (0.1 mmol, 0.1 eq. $), \mathrm{MeCN}(6.0 \mathrm{~mL}), 80^{\circ} \mathrm{C}$.

\section{Discussion}

Based on our previous work on the synthesis and application of differentiated di-boron compounds [18], we applied this new strategy to the preparation of di-boron reagents containing the $\mathrm{B}(\mathrm{dan})$ group. Taking the di-functionalized compound $\mathbf{1} \mathbf{j}$ as an example (Scheme 3 ), the potential application of this product was checked (The details were shown in Supplementary). Firstly, in the presence of $\mathrm{Pd}(\mathrm{II})$ catalyst, the $\mathrm{Br}$ atom could react with $\mathrm{B}_{2}(\mathrm{pin})_{2}$ to synthesize the site-differentiated diboron arene 2. As anticipated $[9,14]$, the reactivities of $B($ pin) and $B($ dan), with two effective masking groups for boronic acids on 2, could be differentiated in the Suzuki-Miyaura coupling reaction. The $\mathrm{B}(\mathrm{pin})$ group could be selectively transformed into the aryl while the $\mathrm{B}(\mathrm{dan})$ group remained intact. After the workup and purification process, the compound 3 was obtained in $81 \%$ yield. In agreement with previous experience, the $\mathrm{B}(\mathrm{dan})$ group on compound 3 could be activated by hydrolysis under aqueous condition to obtain its boronic acid, which can be used in the sequential Suzuki-Miyaura cross-coupling reaction to form compound 4 . Therefore, we have demonstrated the usefulness of the naphthalene-1,8-diamino borylation in the facile preparation of boron-differentiated di-boron compounds, which may serve as polyvalent nucleophiles for the modular construction of multifunctionalized poly(hetero)arenes by consecutive cross coupling reactions. 


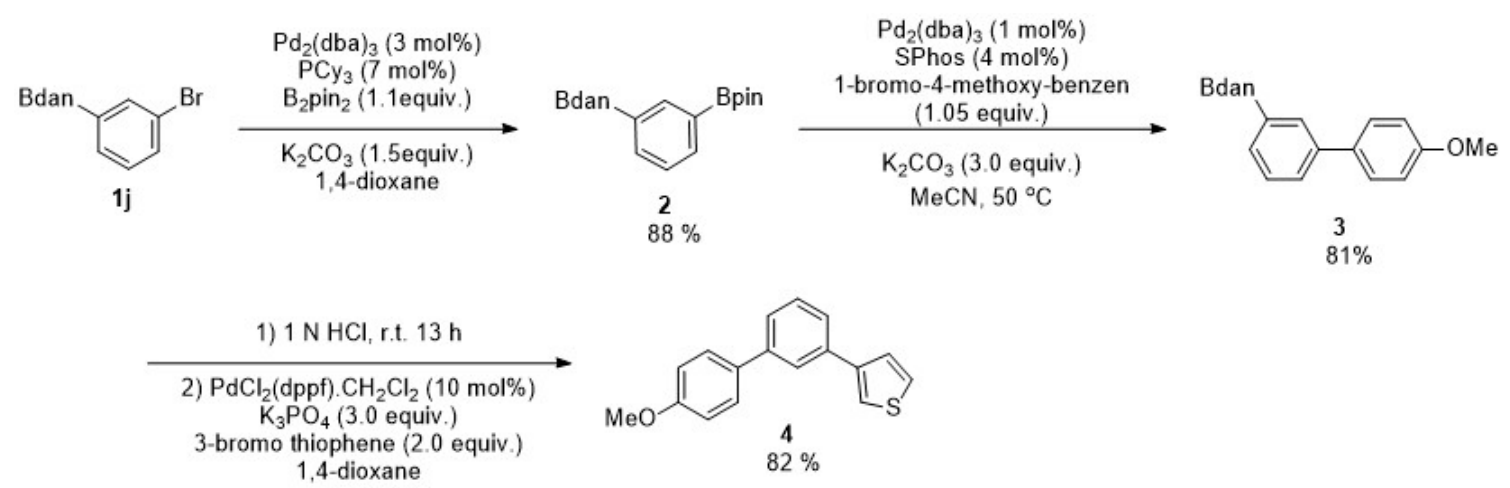

Scheme 3. Successive and selective Suzuki-Miyaura cross-coupling reaction using 3-bromophenyl $\mathrm{B}$ (dan) $\mathbf{2} \mathbf{j}$ as the starting material.

\section{Materials and Methods}

\subsection{Methods and Material}

\subsubsection{General Information}

Unless otherwise noted, all reactions were carried out in a flame-dried, sealed Schlenk reaction tube under an atmosphere of nitrogen. Analytical thin-layer chromatography (TLC) was performed on glass plates coated with $0.25 \mathrm{~mm} 230-400$ mesh silica gel containing a fluorescent indicator. Preparative thin-layer chromatography (PTLC) was performed on pre-coated, glass-backed GF254 silica gel plates. Visualization was accomplished by exposure to a UV lamp. All the products in this article are compatible with standard silica gel chromatography. Column chromatography was performed on silica gel (200-300 mesh) using standard methods.

\subsubsection{Structural Analysis}

NMR spectra were measured on a nuclear magnetic resonance apparatus (Avance III HD 400M, Bruker, Germany) and chemical shifts $(\delta)$ are reported in parts per million (ppm). ${ }^{1} \mathrm{H}-\mathrm{NMR}$ spectra were recorded at $400 \mathrm{MHz}$ in NMR solvents and referenced internally to corresponding solvent resonance, and ${ }^{13} \mathrm{C}-\mathrm{NMR}$ spectra were recorded at $100 \mathrm{MHz}$ and referenced to corresponding solvent resonance. Carbons bearing boron substituents were generally not observed due to quadrupolar relaxation. Coupling constants are reported in $\mathrm{Hz}$ with multiplicities denoted as s (singlet), $\mathrm{d}$ (doublet), $\mathrm{t}$ (triplet), q (quartet), $\mathrm{m}$ (multiplet) and br (broad). Infrared spectra were collected on a Thermo Fisher Nicolet 6700 FT-IR spectrometer (Waltham, MA, USA) using ATR (Attenuated Total Reflectance) method. Absorption maxima $\left(v\right.$ max) are reported in wavenumbers $\left(\mathrm{cm}^{-1}\right)$. High resolution mass spectra (HRMS) were acquired with an ESI source or APCI source (MTQ III q-TOP, Bruker, Germany).

\subsubsection{Materials}

Commercial reagents and solvent were purchased from J\&K, Energy, Sigma-Aldrich, Alfa Aesar, Acros Organics, Strem Chemicals, TCI and used as received unless otherwise stated.

\subsection{General Procedure for the Direct Transformation from Arylamines to Aryl Naphthalene-1,8- diamino Boronamides}

In air, Bpin-B(dan) (0.1 mmol, 1.0 eq.), aryl amide (0.2 mmol, 2.0 eq.), TBAI (0.01 eq.), $\mathrm{NaOAc}(0.15$ eq.), and BPO (0.01 eq.) were sequentially weighed and added to a screw-capped Schenk tube containing a magnetic stir bar. The vessel was evacuated and refilled with nitrogen for three times. $t$-BuONO (0.2 eq.) and $\mathrm{MeCN}(0.6 \mathrm{~mL})$ were added in turn under $\mathrm{N}_{2}$ atmosphere using syringes through a septum which was temporarily used to replace the screw cap. The reaction mixture was then vigorously stirred at $80{ }^{\circ} \mathrm{C}$ for the indicated time. The resulting mixture was filtered through 
a pad of Celite ${ }^{\circledR}$, and the filter cake was washed with ethyl acetate $(3 \mathrm{~mL} \times 2)$. The combined filtrate was evaporated under vacuum to dryness and the residue was purified by column chromatography to yield the desired product.

\subsection{Analytical Data of Products $\mathbf{1 a}-\mathbf{1 t}$}

2-(4-methoxyphenyl)-2,3-dihydro-1H-naphtho[1,8-de][1,3,2]diazaborinine (1a, CAS: 1159803-53-8) [25]. Yield: $20.6 \mathrm{mg}$ (75\%); white solid; m.p.: $163.2 \sim 165.6^{\circ} \mathrm{C}$; IR $\left(\mathrm{cm}^{-1}\right)$ : 3407, 1594, 1495, 1407, 1224, 1181, 1029; ${ }^{1} \mathrm{H}-\mathrm{NMR}\left(400 \mathrm{MHz}, \mathrm{CDCl}_{3}\right) \delta 7.59(\mathrm{~d}, J=8.8 \mathrm{~Hz}, 2 \mathrm{H}), 7.15(\mathrm{t}, J=8.0 \mathrm{~Hz}, 2 \mathrm{H}), 7.05(\mathrm{~d}, J=8.0 \mathrm{~Hz}$, $2 \mathrm{H}), 6.98(\mathrm{~d}, J=8.4 \mathrm{~Hz}, 2 \mathrm{H}), 6.42(\mathrm{~d}, J=7.2 \mathrm{~Hz}, 2 \mathrm{H}), 6.00(\mathrm{~s}, 2 \mathrm{H}), 3.86(\mathrm{~s}, 3 \mathrm{H}) ;{ }^{13} \mathrm{C}-\mathrm{NMR}(100 \mathrm{MHz}$, $\left.\mathrm{CDCl}_{3}\right) \delta 161.4,141.2,140.3,136.4,133.0,127.6,117.7,113.9,105.9,55.2 ;{ }^{11} \mathrm{~B}-\mathrm{NMR}\left(128 \mathrm{MHz}, \mathrm{CDCl}_{3}\right) \delta$ 29.1; HRMS (APCI) $m / z$ calcd for $\mathrm{C}_{17} \mathrm{H}_{14} \mathrm{BN}_{2} \mathrm{O}\left(\mathrm{M}^{-}\right)$: 273.1205, found: 273.1203 .

2-(4-bromophenyl)-2,3-dihydro-1H-naphtho[1,8-de][1,3,2]diazaborinine (1b) [9]. Yield: $20.0 \mathrm{mg}(62 \%)$; white solid; m.p.: $135.6 \sim 136.2^{\circ} \mathrm{C}$; IR $\left(\mathrm{cm}^{-1}\right)$ : 3408.9, 2921.3, 2851.4, 2362.0, 2342.0, 1596.0, 1511.4, 1400.3, 1373.3, 817.5, 752.2, 690.0; ${ }^{1} \mathrm{H}-\mathrm{NMR}\left(400 \mathrm{MHz}, \mathrm{CDCl}_{3}\right) \delta 7.57(\mathrm{~d}, J=8.2 \mathrm{~Hz}, 2 \mathrm{H}), 7.48(\mathrm{~d}, J=8.2 \mathrm{~Hz}$, $2 \mathrm{H}), 7.14(\mathrm{t}, J=7.8 \mathrm{~Hz}, 2 \mathrm{H}), 7.06(\mathrm{~d}, J=8.0 \mathrm{~Hz}, 2 \mathrm{H}), 6.40(\mathrm{~d}, J=7.2 \mathrm{~Hz}, 2 \mathrm{H}), 5.96(\mathrm{~s}, 2 \mathrm{H}) ;{ }^{13} \mathrm{C}-\mathrm{NMR}$ $\left(100 \mathrm{MHz}, \mathrm{CDCl}_{3}\right) \delta 140.8,136.3,133.0,131.5,127.7,124.9,119.9,118.1,106.2 ;$ HRMS (APCI) $m / z$ calcd for $\mathrm{C}_{16} \mathrm{H}_{12} \mathrm{BBrN}_{2}\left(\mathrm{M}^{-}\right)$: 322.0282 , found: 322.0279 .

2-(4-(trifluoromethyl)phenyl)-2,3-dihydro-1H-naphtho[1,8-de][1,3,2]diazaborinine (1c) [25]. Yield: $23.7 \mathrm{mg}(76 \%)$; white solid; m.p.: $127.0 \sim 130.3{ }^{\circ} \mathrm{C}$; IR $\left(\mathrm{cm}^{-1}\right)$ : 3414.2, 2365.0, 2341.9, 1527.7, 1493.5, 1398.9, 1166.0, 1035.0, 826.8, 747.1; ${ }^{1} \mathrm{H}-\mathrm{NMR}\left(400 \mathrm{MHz}, \mathrm{CDCl}_{3}\right) \delta 7.75(\mathrm{~d}, J=7.9 \mathrm{~Hz}, 2 \mathrm{H}), 7.69(\mathrm{~d}$, $J=8.0 \mathrm{~Hz}, 2 \mathrm{H}), 7.13(\mathrm{t}, J=7.8 \mathrm{~Hz}, 2 \mathrm{H}), 7.09(\mathrm{~d}, J=7.8 \mathrm{~Hz}, 2 \mathrm{H}), 6.44(\mathrm{dd}, J=7.2,0.8 \mathrm{~Hz}, 2 \mathrm{H}), 6.01(\mathrm{~s}, 2 \mathrm{H})$; ${ }^{13} \mathrm{C}-\mathrm{NMR}\left(100 \mathrm{MHz}, \mathrm{CDCl}_{3}\right) \delta 140.8,136.5,132.2(\mathrm{q}, J=32 \mathrm{~Hz}), 131.9,127.8,125.1(\mathrm{q}, J=4 \mathrm{~Hz}), 124.2$ $(\mathrm{q}, J=272 \mathrm{~Hz}), 120.1,118.4,103.8 ;{ }^{11} \mathrm{~B}-\mathrm{NMR}\left(128 \mathrm{MHz}, \mathrm{CDCl}_{3}\right) \delta 30.1 ;{ }^{19} \mathrm{~F}-\mathrm{NMR}\left(377 \mathrm{MHz}, \mathrm{CDCl}_{3}\right) \delta$ -62.88; HRMS (APCI) $m / z$ calcd for $\mathrm{C}_{17} \mathrm{H}_{11} \mathrm{BF}_{3} \mathrm{~N}_{2}\left(\mathrm{M}^{-}\right)$: 311.0973, found: 311.0974.

4-(1H-naphtho[1,8-de][1,3,2]diazaborinin-2(3H)-yl)phenol (1d) [25]. Yield: $13.5 \mathrm{mg}$ (52\%); white solid; m.p.: $222.6 \sim 225.7^{\circ} \mathrm{C}$; IR $\left(\mathrm{cm}^{-1}\right)$ : 3441.0, 3414.9, 3028.7, 2361.5, 2343.2, 1582.3, 1487.1, 1404.1, 1200.2, $1177.8,813.5,753.2 ;{ }^{1} \mathrm{H}-\mathrm{NMR}\left(400 \mathrm{MHz}, \mathrm{CDCl}_{3}\right) \delta 7.55(\mathrm{~d}, J=8.2 \mathrm{~Hz}, 2 \mathrm{H}), 7.14(\mathrm{t}, J=7.8 \mathrm{~Hz}, 2 \mathrm{H}), 7.05(\mathrm{~d}$, $J=8.2 \mathrm{~Hz}, 2 \mathrm{H}), 6.91(\mathrm{~d}, J=8.3 \mathrm{~Hz}, 2 \mathrm{H}), 6.41(\mathrm{~d}, J=7.3 \mathrm{~Hz}, 2 \mathrm{H}), 5.98(\mathrm{~s}, 2 \mathrm{H}), 4.90(\mathrm{~s}, 1 \mathrm{H}) ;{ }^{13} \mathrm{C}-\mathrm{NMR}$ $\left(100 \mathrm{MHz}, \mathrm{CDCl}_{3}\right) \delta 157.4,141.2,136.3,133.3,127.6,119.7,117.7,115.3,105.9 ;$ HRMS (APCI) $\mathrm{m} / z$ calcd for $\mathrm{C}_{16} \mathrm{H}_{12} \mathrm{BN}_{2} \mathrm{O}\left(\mathrm{M}^{-}\right)$: 259.1048, found: 259.1049.

4-(1H-naphtho[1,8-de][1,3,2]diazaborinin-2(3H)-yl)benzonitrile (1e) [25]. Yield: $13.7 \mathrm{mg}(51 \%)$; white solid; m.p.: $220.4 \sim 225.1{ }^{\circ} \mathrm{C}$; IR $\left(\mathrm{cm}^{-1}\right)$ : 3409.3, 3401.7, 3393.0, 3370.2, 2362.0, 2220.1, 1516.3, 1593.4, 1405.2, 1082.6, 818.4; ${ }^{1} \mathrm{H}-\mathrm{NMR}\left(400 \mathrm{MHz}, \mathrm{CDCl}_{3}\right) \delta 7.72(\mathrm{dd}, J=8.0,3.2 \mathrm{~Hz}, 4 \mathrm{H}), 7.14(\mathrm{t}$, $J=7.7 \mathrm{~Hz}, 2 \mathrm{H}), 7.09(\mathrm{~d}, J=8.0 \mathrm{~Hz}, 2 \mathrm{H}), 6.43(\mathrm{~d}, J=7.2 \mathrm{~Hz}, 2 \mathrm{H}), 5.99(\mathrm{~s}, 2 \mathrm{H}) ;{ }^{13} \mathrm{C}-\mathrm{NMR}(100 \mathrm{MHz}$, $\left.\mathrm{CDCl}_{3}\right) \delta 140.4,136.3,132.0,131.7,127.7,120.0,118.7,118.5,113.7,106.4 ;{ }^{11} \mathrm{~B}-\mathrm{NMR}\left(128 \mathrm{MHz}_{,} \mathrm{CDCl}_{3}\right) \delta$ 28.8; HRMS (ESI) $m / z$ calcd for $\mathrm{C}_{17} \mathrm{H}_{12} \mathrm{BN}_{3} \mathrm{Na}\left(\mathrm{M}^{+}\right)$: 292.1022, found: 292.1014 .

methyl 4-(1H-naphtho[1,8-de][1,3,2]diazaborinin-2(3H)-yl)benzoate (1f). Yield: $24.5 \mathrm{mg}(81 \%)$; white solid; m.p.: $202.2 \sim 203.6^{\circ} \mathrm{C}$; IR $\left(\mathrm{cm}^{-1}\right)$ : 3387.9, 2920.4, 2849.8, 2364.4, 2342.4, 1705.0, 1592.7, 1397.3, 759.4, 704.7; ${ }^{1} \mathrm{H}-\mathrm{NMR}\left(400 \mathrm{MHz}, \mathrm{CDCl}_{3}\right) \delta 8.09(\mathrm{~d}, J=8.0 \mathrm{~Hz}, 2 \mathrm{H}), 7.71(\mathrm{~d}, J=7.9 \mathrm{~Hz}, 2 \mathrm{H}), 7.15$ $(\mathrm{t}, J=7.8 \mathrm{~Hz}, 2 \mathrm{H}), 7.07(\mathrm{~d}, J=8.2 \mathrm{~Hz}, 2 \mathrm{H}), 6.43(\mathrm{~d}, J=7.2 \mathrm{~Hz}, 2 \mathrm{H}), 6.05(\mathrm{~s}, 2 \mathrm{H}) .3 .95(\mathrm{~s}, 3 \mathrm{H}) ;{ }^{13} \mathrm{C}-\mathrm{NMR}$ $\left(100 \mathrm{MHz}, \mathrm{CDCl}_{3}\right) \delta 167.0,140.8,136.4,131.6,131.5,129.2,127.6,120.0,118.2,106.2,52.3 ;$ HRMS (APCI) $m / z$ calcd for $\mathrm{C}_{18} \mathrm{H}_{15} \mathrm{BN}_{2} \mathrm{O}_{2}\left(\mathrm{M}^{-}\right)$: 302.1232, found: 302.1230 . 
2-(m-tolyl)-2,3-dihydro-1H-naphtho[1,8-de][1,3,2]diazaborinine (1g) [25]. Yield: $18.3 \mathrm{mg}(71 \%)$; white solid; m.p.: $103.8 \sim 106.3{ }^{\circ} \mathrm{C}$; IR $\left(\mathrm{cm}^{-1}\right)$ : 3409.1, 3050.8, 3029.9, 1593.0, 1581.1, 1326.2, 817.9, 762.0, 702.8; ${ }^{1} \mathrm{H}-\mathrm{NMR}\left(400 \mathrm{MHz}, \mathrm{CDCl}_{3}\right) \delta 7.44(\mathrm{~d}, J=8.2 \mathrm{~Hz}, 2 \mathrm{H}), 7.34(\mathrm{~m}, 2 \mathrm{H}), 7.14(\mathrm{t}, J=7.8 \mathrm{~Hz}, 2 \mathrm{H})$, $7.05(\mathrm{~d}, J=8.2 \mathrm{~Hz}, 2 \mathrm{H}), 6.41(\mathrm{~d}, J=7.3 \mathrm{~Hz}, 2 \mathrm{H}), 6.02(\mathrm{~s}, 2 \mathrm{H}), 2.42(\mathrm{~s}, 3 \mathrm{H}) ;{ }^{13} \mathrm{C}-\mathrm{NMR}\left(100 \mathrm{MHz}, \mathrm{CDCl}_{3}\right) \delta$ 141.2, 137.7, 136.4, 132.2, 131.1, 128.5, 128.2, 127.6, 119.9, 117.8, 106.0, 21.6; ${ }^{11} \mathrm{~B}$ NMR (128 MHz, $\left.\mathrm{CDCl}_{3}\right)$ $\delta$ 30.4; HRMS (APCI) $m / z$ calcd for $\mathrm{C}_{17} \mathrm{H}_{14} \mathrm{BN}_{2}\left(\mathrm{M}^{-}\right)$: 257.1256, found: 257.1257.

2-(3-methoxyphenyl)-2,3-dihydro-1H-naphtho[1,8-de][1,3,2]diazaborinine (1h) [25]. Yield: $19.2 \mathrm{mg}$ (70\%); white solid; m.p.: $113.7 \sim 116.4{ }^{\circ} \mathrm{C}$; IR $\left(\mathrm{cm}^{-1}\right)$ : 3452.9, 3409.8, 3049.7, 3032.7, 1593.0, 1515.4, 1478.6, 1406.1, 1243.3, 693.5, 658.1; ${ }^{1} \mathrm{H}-\mathrm{NMR}\left(400 \mathrm{MHz}, \mathrm{CDCl}_{3}\right) \delta 7.37(\mathrm{t}, J=7.7 \mathrm{~Hz}, 1 \mathrm{H}), 7.21(\mathrm{~d}, J=7.2 \mathrm{~Hz}, 1 \mathrm{H})$, $7.14(\mathrm{~m}, 3 \mathrm{H}), 7.05(\mathrm{~d}, J=8.1 \mathrm{~Hz}, 2 \mathrm{H}), 7.00(\mathrm{dd}, J=8.1,2.0 \mathrm{~Hz}, 1 \mathrm{H}), 6.40(\mathrm{~d}, J=7.3 \mathrm{~Hz}, 2 \mathrm{H}), 6.00(\mathrm{~s}, 2 \mathrm{H})$, $3.86(\mathrm{~s}, 3 \mathrm{H}) ;{ }^{13} \mathrm{C}-\mathrm{NMR}\left(100 \mathrm{MHz}, \mathrm{CDCl}_{3}\right) \delta 159.5,141.1,136.4,129.6,127.7,123.9,119.9,117.9,117.0$, 115.5, 106.1, 55.3; ${ }^{11} \mathrm{~B}-\mathrm{NMR}\left(128 \mathrm{MHz}, \mathrm{CDCl}_{3}\right) \delta 30.1$; HRMS (APCI) $m / z$ calcd for $\mathrm{C}_{17} \mathrm{H}_{14} \mathrm{BN}_{2} \mathrm{O}\left(\mathrm{M}^{-}\right.$): 273.1205, found: 273.1207 .

2-(3-(trifluoromethoxy)phenyl)-2,3-dihydro-1H-naphtho[1,8-de][1,3,2]diazaborinine (1i). Yield: $21.3 \mathrm{mg}(65 \%)$; brown oil; IR $\left(\mathrm{cm}^{-1}\right)$ : 3414.2, 2365.0, 2341.9, 1527.7, 1493.5, 1398.9, 1166.0, 1035.0, 826.8, 747.1; ${ }^{1} \mathrm{H}-\mathrm{NMR}\left(400 \mathrm{MHz}, \mathrm{CDCl}_{3}\right) \delta 7.56(\mathrm{~d}, J=7.4 \mathrm{~Hz}, 2 \mathrm{H}), 7.48(\mathrm{~m}, 2 \mathrm{H}), 7.33(\mathrm{~d}, J=8.1 \mathrm{~Hz}, 1 \mathrm{H})$, $7.16(\mathrm{t}, J=7.7 \mathrm{~Hz}, 2 \mathrm{H}), 7.08(\mathrm{~d}, J=8.2 \mathrm{~Hz}, 2 \mathrm{H}), 6.43(\mathrm{~d}, J=7.2 \mathrm{~Hz}, 2 \mathrm{H}), 5.98(\mathrm{~s}, 2 \mathrm{H}) ;{ }^{13} \mathrm{C}-\mathrm{NMR}(100 \mathrm{MHz}$, $\left.\mathrm{CDCl}_{3}\right) \delta 149.4,140.7,136.3,129.8,127.7,123.7,122.7,119.9,118.2,106.1$; HRMS (APCI) $\mathrm{m} / z$ calcd for $\mathrm{C}_{17} \mathrm{H}_{12} \mathrm{BF}_{3} \mathrm{~N}_{2} \mathrm{O}\left(\mathrm{M}^{-}\right)$: 328.1000, found: 328.1002 .

2-(3-bromophenyl)-2,3-dihydro-1H-naphtho[1,8-de][1,3,2]diazaborinine (1j) [9]. Yield: $19.3 \mathrm{mg}$ (60\%); white solid; m.p.: $86.6 \sim 87.9^{\circ} \mathrm{C}$; IR $\left(\mathrm{cm}^{-1}\right)$ : 3408.9, 2921.3, 2851.4, 2362.0, 2342.0, 1596.0, 1511.4, 1400.3, 1373.3, 817.5, 752.2, 690.0; ${ }^{1} \mathrm{H}-\mathrm{NMR}\left(400 \mathrm{MHz}, \mathrm{CDCl}_{3}\right) \delta 7.73(\mathrm{~s}, 1 \mathrm{H}), 7.57(\mathrm{~d}, J=8.0 \mathrm{~Hz}, 1 \mathrm{H}), 7.51(\mathrm{~d}$, $J=7.4 \mathrm{~Hz}, 1 \mathrm{H}), 7.29(\mathrm{t}, J=7.7 \mathrm{~Hz}, 1 \mathrm{H}), 7.13(\mathrm{t}, J=7.8 \mathrm{~Hz}, 2 \mathrm{H}), 7.05(\mathrm{~d}, J=7.9 \mathrm{~Hz}, 2 \mathrm{H}), 6.39(\mathrm{~d}, J=7.2$ $\mathrm{Hz}, 2 \mathrm{H}), 5.94(\mathrm{~s}, 2 \mathrm{H}) ;{ }^{13} \mathrm{C}-\mathrm{NMR}\left(100 \mathrm{MHz}, \mathrm{CDCl}_{3}\right) \delta 140.7,136.3,134.4,133.2,130.1,129.9,127.7,123.1$, $119.9,118.1,106.3$; HRMS (APCI) $m / z$ calcd for $\mathrm{C}_{16} \mathrm{H}_{12} \mathrm{BBrN}_{2}\left(\mathrm{M}^{-}\right)$: 322.0282 , found: 322.0280 .

2-(3-fluorophenyl)-2,3-dihydro-1H-naphtho[1,8-de][1,3,2]diazaborinine (1k). Yield: $16.8 \mathrm{mg}(64 \%)$; yellow solid; m.p.: $103.3 \sim 104.6^{\circ} \mathrm{C}$; IR $\left(\mathrm{cm}^{-1}\right)$ : 3442.7, 3435.4, 3032.2, 1595.8, 1520.5, 1371.4, 757.1, 749.4, 686.3; ${ }^{1} \mathrm{H}-\mathrm{NMR}\left(400 \mathrm{MHz}, \mathrm{CDCl}_{3}\right) \delta 7.41(\mathrm{~m}, 2 \mathrm{H}), 7.33(\mathrm{dd}, J=2.4,9.2 \mathrm{~Hz}, 1 \mathrm{H}), 7.14(\mathrm{~m}, 3 \mathrm{H}), 7.06(\mathrm{~d}$, $J=8.0 \mathrm{~Hz}, 2 \mathrm{H}), 6.41(\mathrm{~d}, J=7.3 \mathrm{~Hz}, 2 \mathrm{H}), 5.97(\mathrm{~s}, 2 \mathrm{H}) ;{ }^{13} \mathrm{C}-\mathrm{NMR}\left(100 \mathrm{MHz}, \mathrm{CDCl}_{3}\right) \delta 162.9(\mathrm{~d}, J=246$ $\mathrm{Hz}), 140.8,136.3,130.1(\mathrm{~d}, J=7 \mathrm{~Hz}), 127.7,127.0(\mathrm{~d}, J=3 \mathrm{~Hz}), 119.9,118.1,118.0(\mathrm{~d}, J=23 \mathrm{~Hz}), 117.1(\mathrm{~d}$, $J=20 \mathrm{~Hz}$ ), 106.2; HRMS (APCI) $m / z$ calcd for $\mathrm{C}_{16} \mathrm{H}_{12} \mathrm{BFN}_{2}\left(\mathrm{M}^{-}\right)$: 262.1083, found: 262.1080 .

Methyl 3-(1H-naphtho[1,8-de][1,3,2]diazaborinin-2(3H)-yl)benzoate (11). Yield: $23.6 \mathrm{mg}$ (78\%); pink solid; m.p.: 178.9 179.8 ${ }^{\circ} \mathrm{C}$; IR $\left(\mathrm{cm}^{-1}\right)$ : 3452.9, 3409.8, 2049.7, 1515.4, 1478.6, 1077.0, 816.1; ${ }^{1} \mathrm{H}-\mathrm{NMR}\left(400 \mathrm{MHz}, \mathrm{CDCl}_{3}\right) \delta 8.33(\mathrm{~s}, 1 \mathrm{H}), 8.13(\mathrm{dt}, J=8.8,1.4 \mathrm{~Hz}, 1 \mathrm{H}), 7.83(\mathrm{~d}, J=7.4 \mathrm{~Hz}, 1 \mathrm{H}), 7.52(\mathrm{t}$, $J=7.6 \mathrm{~Hz}, 1 \mathrm{H}), 7.15(\mathrm{t}, J=7.8 \mathrm{~Hz}, 2 \mathrm{H}), 7.07(\mathrm{~d}, J=8.0 \mathrm{~Hz}, 2 \mathrm{H}), 6.45(\mathrm{~d}, J=7.2 \mathrm{~Hz}, 2 \mathrm{H}), 6.09(\mathrm{~s}, 2 \mathrm{H}), 3.97$ $(\mathrm{s}, 3 \mathrm{H}) ;{ }^{13} \mathrm{C}-\mathrm{NMR}\left(100 \mathrm{MHz}, \mathrm{CDCl}_{3}\right) \delta 167.2,140.8,136.3,135.9,132.6,131.3,130.0,128.4,127.7,119.9$, 118.1, 106.2, 52.3; HRMS (APCI) $\mathrm{m} / z$ calcd for $\mathrm{C}_{18} \mathrm{H}_{15} \mathrm{BN}_{2} \mathrm{O}_{2}\left(\mathrm{M}^{-}\right)$: 302.1232, found: 302.1228.

3-(1H-naphtho[1,8-de][1,3,2]diazaborinin-2(3H)-yl)benzamide (1m). Yield: $15.5 \mathrm{mg}$ (54\%); brown solid; m.p.: 168.4 169.5 ${ }^{\circ} \mathrm{C}$; IR $\left(\mathrm{cm}^{-1}\right)$ : 3382.8, 3101.1, 3048.7, 2341.2, 2366.2, 1599.3, 1568.7, 1506.6, 816.3, 758.5, 668.6; ${ }^{1} \mathrm{H}-\mathrm{NMR}\left(400 \mathrm{MHz}, \mathrm{CDCl}_{3}\right) \delta 7.85(\mathrm{~s}, 1 \mathrm{H}), 7.49(\mathrm{~m}, 1 \mathrm{H}), 7.36(\mathrm{~m}, 3 \mathrm{H}), 7.30(\mathrm{~s}, 1 \mathrm{H}), 7.13$ $(\mathrm{t}, J=7.7 \mathrm{~Hz}, 2 \mathrm{H}), 7.05(\mathrm{~d}, J=8.2 \mathrm{~Hz}, 2 \mathrm{H}), 6.39$ (d, $J=7.0 \mathrm{~Hz}, 2 \mathrm{H}), 6.05(\mathrm{~s}, 2 \mathrm{H}) ;{ }^{13} \mathrm{C}-\mathrm{NMR}(100 \mathrm{MHz}$, $\mathrm{CDCl}_{3}$ ) $\delta 168.6,141.0,137.8,136.3,129.0,127.6,127.5,122.9,121.7,119.9,117.8,106.1$; HRMS (APCI) $m / z$ calcd for $\mathrm{C}_{17} \mathrm{H}_{14} \mathrm{BN}_{3} \mathrm{O}\left(\mathrm{M}^{-}\right)$: 287.1235, found: 287.1233 . 
2-(3,4-dimethoxyphenyl)-2,3-dihydro-1H-naphtho[1,8-de][1,3,2]diazaborinine (1n). Yield: $18.8 \mathrm{mg}$ (62\%); light pink solid; m.p.: $174.5 \sim 175.0^{\circ} \mathrm{C}$; IR $\left(\mathrm{cm}^{-1}\right)$ : 3407.2, 1594.4, 1495.9, 1407.7, 1224.1, 1181.2, 1029.0; ${ }^{1} \mathrm{H}-\mathrm{NMR}\left(400 \mathrm{MHz}, \mathrm{CDCl}_{3}\right) \delta 7.24(\mathrm{~d}, J=7.9 \mathrm{~Hz}, 1 \mathrm{H}), 7.13(\mathrm{~m}, 3 \mathrm{H}), 7.06(\mathrm{~d}, J=8.2 \mathrm{~Hz}, 2 \mathrm{H}), 6.96$ $(\mathrm{d}, J=7.9 \mathrm{~Hz}, 1 \mathrm{H}), 6.43(\mathrm{~d}, J=7.2 \mathrm{~Hz}, 2 \mathrm{H}), 5.99(\mathrm{~s}, 2 \mathrm{H}), 3.97(\mathrm{~s}, 3 \mathrm{H}), 3.93(\mathrm{~s}, 3 \mathrm{H}) ;{ }^{13} \mathrm{C}-\mathrm{NMR}(100 \mathrm{MHz}$, CDCl3) $\delta 150.9,148.9,141.1,136.4,127.6,124.9,119.7,117.8,113.8,111.1,106.0,56.1,55.8 ;$ HRMS (APCI) $m / z$ calcd for $\mathrm{C}_{18} \mathrm{H}_{17} \mathrm{BN}_{2} \mathrm{O}_{2}\left(\mathrm{M}^{-}\right)$: 304.1389, found: 304.1385 .

2-(benzo[d][1,3]dioxol-5-yl)-2,3-dihydro-1H-naphtho[1,8-de][1,3,2]diazaborinine (1o) [25]. Yield: $19.9 \mathrm{mg}(69 \%)$; white solid; m.p.: $173.5 \sim 175.8^{\circ} \mathrm{C}$; IR $\left(\mathrm{cm}^{-1}\right)$ : 3399.2, 1594.3, 1478.2, 1402.7, 1232.1, 1034.0; ${ }^{1} \mathrm{H}-\mathrm{NMR}\left(400 \mathrm{MHz}, \mathrm{CDCl}_{3}\right) \delta 7.15(\mathrm{t}, J=7.4 \mathrm{~Hz}, 3 \mathrm{H}), 7.06(\mathrm{t}, J=6.6 \mathrm{~Hz}, 3 \mathrm{H}), 6.91(\mathrm{~d}, J=7.6 \mathrm{~Hz}$, $1 \mathrm{H}), 6.40(\mathrm{~d}, J=7.2 \mathrm{~Hz}, 2 \mathrm{H}), 6.00(\mathrm{~s}, 2 \mathrm{H}), 5.94(\mathrm{~s}, 2 \mathrm{H}) ;{ }^{13} \mathrm{C}-\mathrm{NMR}\left(100 \mathrm{MHz}, \mathrm{CDCl}_{3}\right) \delta 149.4,147.8,141.1$, 136.3, 127.6, 125.8, 119.7, 117.8, 110.9, 108.8, 106.0, 101.0; HRMS (ESI) $m / z$ calcd for $\mathrm{C}_{17} \mathrm{H}_{14} \mathrm{BN}_{2} \mathrm{O}_{2}\left(\mathrm{M}^{+}\right)$: 289.1148, found: 289.1147 .

2-(3,5-difluorophenyl)-2,3-dihydro-1H-naphtho[1,8-de][1,3,2]diazaborinine (1p). Yield: $17.9 \mathrm{mg}(64 \%)$; yellow solid; m.p.: $113.2 \sim 114.6{ }^{\circ} \mathrm{C}$; IR $\left(\mathrm{cm}^{-1}\right)$ : 3414.2, 2365.0, 2341.9, 1398.8, 1317.8, 1105.6, 1082.3, 818.4; ${ }^{1} \mathrm{H}-\mathrm{NMR}\left(400 \mathrm{MHz}, \mathrm{CDCl}_{3}\right) \delta 7.75(\mathrm{~d}, J=7.9 \mathrm{~Hz}, 2 \mathrm{H}), 7.68(\mathrm{~d}, J=8.0 \mathrm{~Hz}, 2 \mathrm{H}), 7.16(\mathrm{t}, J=7.8 \mathrm{~Hz}$, $2 \mathrm{H}), 7.08(\mathrm{~d}, J=7.8 \mathrm{~Hz}, 2 \mathrm{H}), 6.43(\mathrm{dd}, J=7.2,0.6 \mathrm{~Hz}, 2 \mathrm{H}), 6.01(\mathrm{~s}, 2 \mathrm{H}) ;{ }^{13} \mathrm{C}-\mathrm{NMR}\left(100 \mathrm{MHz}, \mathrm{CDCl}_{3}\right) \delta$ $160.7(\mathrm{dd}, J=249 \mathrm{~Hz}, 6 \mathrm{~Hz}), 137.9,133.8,125.1,117.4,115.8,111.3(\mathrm{dd}, J=17 \mathrm{~Hz}, 6 \mathrm{~Hz}), 103.8,102.9$ $(\mathrm{t}, J=25 \mathrm{~Hz})$; HRMS (APCI) $m / z$ calcd for $\mathrm{C}_{16} \mathrm{H}_{11} \mathrm{BN}_{2} \mathrm{~F}_{2}\left(\mathrm{M}^{-}\right)$: 280.0989 , found: 280.0991 .

2-(3,5-dichlorophenyl)-2,3-dihydro-1H-naphtho[1,8-de][1,3,2]diazaborinine (1q) [63]. Yield: $20.6 \mathrm{mg}$ (66\%); green solid; m.p.: $164.2 \sim 165.8^{\circ} \mathrm{C}$; IR $\left(\mathrm{cm}^{-1}\right)$ : 3381.9, 2946.7, 2364.1, 1559.7, 1507.0, 1266.1, 1068.1, 746.4; ${ }^{1} \mathrm{H}-\mathrm{NMR}\left(400 \mathrm{MHz}, \mathrm{CDCl}_{3}\right) \delta 7.46(\mathrm{dd}, J=8.3,1.7 \mathrm{~Hz}, 3 \mathrm{H}), 7.15(\mathrm{t}, J=8.0 \mathrm{~Hz}, 2 \mathrm{H}), 7.08(\mathrm{~d}$, $J=8.0 \mathrm{~Hz}, 2 \mathrm{H}), 6.42(\mathrm{~d}, J=7.2 \mathrm{~Hz}, 2 \mathrm{H}), 5.94(\mathrm{~s}, 2 \mathrm{H}) ;{ }^{13} \mathrm{C}-\mathrm{NMR}\left(100 \mathrm{MHz}, \mathrm{CDCl}_{3}\right) \delta$ 140.4, 136.3, 135.3, $130.1,129.7,127.7,120.0,118.4,106.4$; HRMS (APCI) $m / z$ calcd for $\mathrm{C}_{16} \mathrm{H}_{11} \mathrm{BN}_{2} \mathrm{Cl}_{2}\left(\mathrm{M}^{-}\right)$: 312.0398, found: 312.0395 .

2-(o-tolyl)-2,3-dihydro-1H-naphtho[1,8-de][1,3,2]diazaborinine (1r) [25]. Yield: $11.6 \mathrm{mg}(45 \%)$; white solid; m.p.: $73.2 \sim 75.1{ }^{\circ} \mathrm{C}$; IR $\left(\mathrm{cm}^{-1}\right)$ : 3420.1, 3404.9, 2360.8, 2341.0, 1594.1, 1506.1, 1325.8, 1318.1, 1077.9, 818.4, 656.0; ${ }^{1} \mathrm{H}-\mathrm{NMR}\left(400 \mathrm{MHz}, \mathrm{CDCl}_{3}\right) \delta 7.44(\mathrm{~d}, J=7.3 \mathrm{~Hz}, 1 \mathrm{H}), 7.31(\mathrm{t}, J=7.5 \mathrm{~Hz}$, $1 \mathrm{H}), 7.21(\mathrm{~m}, 2 \mathrm{H}), 7.12(\mathrm{t}, J=7.8 \mathrm{~Hz}, 2 \mathrm{H}), 7.05(\mathrm{~d}, J=8.5 \mathrm{~Hz}, 2 \mathrm{H}), 6.33(\mathrm{~d}, J=7.2 \mathrm{~Hz}, 2 \mathrm{H}), 5.80(\mathrm{~s}, 2 \mathrm{H})$, 2.49 (s, 3H); ${ }^{13} \mathrm{C}-\mathrm{NMR}\left(100 \mathrm{MHz}, \mathrm{CDCl}_{3}\right) \delta 141.1,140.7,136.4,132.3,129.7,129.3,127.7,125.3,119.8$, $117.9,105.9,22.4 ;{ }^{11} \mathrm{~B}$ NMR $\left(128 \mathrm{MHz}, \mathrm{CDCl}_{3}\right) \delta 30.0$; HRMS (APCI) $m / z$ calcd for $\mathrm{C}_{17} \mathrm{H}_{14} \mathrm{BN}_{2}\left(\mathrm{M}^{-}\right)$: 257.1256, found: 257.1257 .

Methyl 2-(1H-naphtho[1,8-de][1,3,2]diazaborinin-2(3H)-yl)benzoate (1s) [25]. Yield: $15.4 \mathrm{mg}$ (51\%); white solid; m.p.: $160.3 \sim 164.5^{\circ} \mathrm{C}$; IR $\left(\mathrm{cm}^{-1}\right)$ : 3381.9, 2946.7, 2364.1, 1699.4, 1507.6, 1134.7, 1266.1, 1068.1, 816.3, 746.4; ${ }^{1} \mathrm{H}-\mathrm{NMR}\left(400 \mathrm{MHz}, \mathrm{CDCl}_{3}\right) \delta 8.04(\mathrm{~d}, J=7.8 \mathrm{~Hz}, 1 \mathrm{H}), 7.54(\mathrm{~m}, 2 \mathrm{H}), 7.47(\mathrm{~m}, 1 \mathrm{H})$, $7.11(\mathrm{t}, J=7.8 \mathrm{~Hz}, 2 \mathrm{H}), 7.04(\mathrm{~d}, J=7.8 \mathrm{~Hz}, 2 \mathrm{H}), 6.31(\mathrm{~d}, J=7.2 \mathrm{~Hz}, 2 \mathrm{H}), 5.73(\mathrm{~s}, 2 \mathrm{H}), 3.86(\mathrm{~s}, 3 \mathrm{H})$; ${ }^{13} \mathrm{C}-\mathrm{NMR}\left(100 \mathrm{MHz}, \mathrm{CDCl}_{3}\right) \delta 168.3,141.3,136.4,133.2,132.7,132.1,129.6,128.9,127.6,119.6,117.6$, 105.8, 52.4; ${ }^{11} \mathrm{~B}$ NMR (128 MHz, $\mathrm{CDCl}_{3}$ ) $\delta$ 31.0; HRMS (APCI) $m / z$ calcd for $\mathrm{C}_{18} \mathrm{H}_{14} \mathrm{BN}_{2} \mathrm{O}_{2}\left(\mathrm{M}^{-}\right.$): 301.1154, found: 301.1157.

2-(naphthalen-1-yl)-2,3-dihydro-1H-naphtho[1,8-de][1,3,2]diazaborinine (2t) [25]. Yield: $14.1 \mathrm{mg}$ (48\%); white solid; m.p.: $140.2 \sim 143.6{ }^{\circ} \mathrm{C}$; IR $\left(\mathrm{cm}^{-1}\right)$ : $3420.2,3402.4,1594.6,1508.8,1498.6,1315.5,1167.3$; ${ }^{1} \mathrm{H}-\mathrm{NMR}\left(400 \mathrm{MHz}, \mathrm{CDCl}_{3}\right) \delta 8.22(\mathrm{dd}, J=6.4,3.2 \mathrm{~Hz}, 1 \mathrm{H}), 7.91(\mathrm{~m}, 2 \mathrm{H}), 7.70(\mathrm{~d}, J=6.4 \mathrm{~Hz}, 1 \mathrm{H}), 7.52$ $(\mathrm{m}, 3 \mathrm{H}), 7.17(\mathrm{t}, J=8.0 \mathrm{~Hz}, 2 \mathrm{H}), 7.11(\mathrm{~d}, J=8.0 \mathrm{~Hz}, 2 \mathrm{H}), 6.38(\mathrm{~d} . J=7.2 \mathrm{~Hz}, 2 \mathrm{H}), 6.02(\mathrm{~s}, 2 \mathrm{H}) ;{ }^{13} \mathrm{C}-\mathrm{NMR}$ $\left(100 \mathrm{MHz}, \mathrm{CDCl}_{3}\right) \delta 141.1,136.4,135.4,133.3,130.7,129.6,128.8,127.9,127.7,126.3,125.9,125.4,120.0$, 118.0, 106.1; ${ }^{11}$ B-NMR (128 MHz, $\left.\mathrm{CDCl}_{3}\right) \delta$ 30.7; HRMS (ESI) $m / z$ calcd for $\mathrm{C}_{20} \mathrm{H}_{16} \mathrm{BN}_{2}\left(\mathrm{M}^{+}\right)$: 295.1407, found: 295.1399 . 


\section{Conclusions}

In conclusion, by employing the non-symmetrical di-boron compound, $\mathrm{B}(\mathrm{pin})-\mathrm{B}(\mathrm{dan})$, as the borylating reagent, we have realized a metal-free Sandmeyer-type borylation reaction under relatively mild conditions and afforded various aryl $\mathrm{B}(\mathrm{dan})$ compounds in moderate yields. The procedure tolerates a series of functional groups, including chloro, bromo, fluoro, ester, hydroxy, cyano and amide groups. Further studies on the mechanism are in progress in our laboratory (The proposed mechanism was shown in the Supporting Information). We anticipate that this protocol described herein could serve as an important supplement to the existing strategies for preparing the aryl-B(dan) compounds and will then find wide application in organic synthesis and related fields.

Supplementary Materials: Experimental procedures and spectral data for the borylated products. This material is available free of charge via the Internet.

Author Contributions: S.D. conceived and designed the experiment; Q.M. and M.Z. performed the experiments; S.D. and H.R. analyzed the data; S.T. and Y.Z. contributed reagents/materials/analysis; S.D. and Z.M. wrote the paper.

Funding: This research was funded by the Special Fund for Talent Research of Xijing University, Grant number XJ17B01, Scientific Research Program Funded by Shaanxi Provincial Education Department (Program No.18JK1196) and the National Natural Science Foundation of Shaanxi, Grant number 2017JQ2037.

Acknowledgments: We would like to thank Pengfei Li ( $\mathrm{Xi}^{\prime}$ an Jiaotong University) very much for his guidance and advices during this work.

Conflicts of Interest: The authors declare no conflict of interest.

\section{References}

1. Hall, D.J. Boronic Acids; Wiley: Weinheim, Germany, 2011.

2. Lennox, A.J.; Lloyd-Jones, G.C. Selection of Boron Reagents for Suzuki-Miyaura Coupling. Chem. Soc. Rev. 2014, 43, 412-443. [CrossRef] [PubMed]

3. Fyfe, J.W.B.; Watson, A.J.B. Recent Developments in Organoboron Chemistry: Old Dogs, New Tricks. Chem 2017, 3, 31-55. [CrossRef]

4. Xu, L.; Zhang, S.; Li, P. Boron-Selective Reactions as Powerful Tools for Modular Synthesis of Diverse Complex Molecules. Chem. Soc. Rev. 2015, 44, 8848-8858. [CrossRef] [PubMed]

5. Neeve, E.C.; Geier, S.J;; Mkhalid, I.A.I.; Westcott, S.A.; Marder, T.B. Diboron(4) Compounds: From Structural Curiosity to Synthetic Workhorse. Chem. Rev. 2016, 116, 9091-9161. [CrossRef] [PubMed]

6. Xu, L.; Wang, G.; Zhang, S.; Wang, H.; Wang, L.; Liu, L.; Jiao, J.; Li, P. Recent Advances in Catalytic C-H Borylation Reactions. Tetrahedron 2017, 73, 7123-7157. [CrossRef]

7. Takaya, J.; Iwasawa, N. Catalytic, Direct Synthesis of Bis(boronate) Compounds. ACS Catal. 2012, 2, 1993-2006. [CrossRef]

8. Xu, L.; Li, P. Differentiated Di- and Polyboron Compounds: Synthesis and Application in Successive Suzuki-Miyaura Coupling. Synlett 2014, 25, 1799-1802. [CrossRef]

9. Noguchi, H.; Hojo, K.; Suginome, M. Boron-Masking Strategy for the Selective Synthesis of Oligoarenes via Iterative Suzuki-Miyaura Coupling. J. Am. Chem. Soc. 2007, 129, 758-759. [CrossRef]

10. Iwadate, N.; Suginome, M. Synthesis of B-Protected $\beta$-Styrylboronic Acids via Iridium-Catalyzed Hydroboration of Alkynes with 1,8-Naphthalenediaminatoborane Leading to Iterative Synthesis of Oligo(phenylenevinylene)s. Org. Lett. 2009, 11, 1899-1902. [CrossRef]

11. Wang, C.; Glorius, F. Controlled Iterative Cross-Coupling: On the Way to the Automation of Organic Synthesis. Angew. Chem. Int. Ed. 2009, 48, 5240-5244. [CrossRef]

12. Gillis, E.P.; Burke, M.D. A Simple and Modular Strategy for Small Molecule Synthesis: Iterative Suzuki-Miyaura Coupling of B-Protected Haloboronic Acid Building Blocks. J. Am. Chem. Soc. 2007, 129, 6716-6717. [CrossRef] [PubMed]

13. Molander, G.A.; Sandrock, D.L. Orthogonal Reactivity in Boryl-Substituted Organotrifluoroborates. J. Am. Chem. Soc. 2008, 130, 15792-15793. [CrossRef] 
14. Noguchi, H.; Shioda, T.; Chou, C.-M.; Suginome, M. Differentially Protected Benzenediboronic Acids: Divalent Cross-Coupling Modules for the Efficient Synthesis of Boron-Substituted Oligoarenes. Org. Lett. 2008, 10, 377-380. [CrossRef] [PubMed]

15. Tobisu, M.; Chatani, N. Devising Boron Reagents for Orthogonal Functionalization through Suzuki-Miyaura Cross-Coupling. Angew. Chem. Int. Ed. 2009, 48, 3565-3568. [CrossRef] [PubMed]

16. Iwadate, N.; Suginome, M. Differentially Protected Diboron for Regioselective Diboration of Alkynes: Internal-Selective Cross-Coupling of 1-Alkene-1,2-diboronic Acid Derivatives. J. Am. Chem. Soc. 2010, 132, 2548-2549. [CrossRef] [PubMed]

17. Hyodo, K.; Suetsugu, M.; Nishihara, Y. Diborylation of Alkynyl MIDA Boronates and Sequential Chemoselective Suzuki-Miyaura Couplings: A Formal Carboborylation of Alkynes. Org. Lett. 2013, 16, 440-443. [CrossRef] [PubMed]

18. Xu, L.; Ding, S.; Li, P. Site-Differentiated Polyboron Arenes Prepared by Direct C-H Borylation and Their Highly Selective Suzuki-Miyaura Cross-Coupling Reactions. Angew. Chem. Int. Ed. 2014, 53, 1822-1826. [CrossRef]

19. Ishiyama, T.; Murata, M.; Miyaura, N. Palladium(0)-Catalyzed Cross-Coupling Reaction of Alkoxydiboron with Haloarenes: A Direct Procedure for Arylboronic Esters. J. Org. Chem. 1995, 60, 7508-7510. [CrossRef]

20. Ishiyama, T.; Itoh, Y.; Kitano, T.; Miyaura, N. Synthesis of Arylboronates via the Palladium(0)-Catalyzed Cross-Coupling Reaction of Tetra(alkoxo)diborons with Aryl Triflates. Tetrahedron Lett. 1997, 38, 3447-3450. [CrossRef]

21. Kleeberg, C.; Dang, L.; Lin, Z.; Marder, T.B. A Facile Route to Aryl Boronates: Room-Temperature, Copper-Catalyzed Borylation of Aryl Halides with Alkoxy Diboron Reagents. Angew. Chem. Int. Ed. 2009, 48, 5350-5354. [CrossRef]

22. Yamamoto, T.; Morita, T.; Takagi, J.; Yamakawa, T. $\mathrm{NiCl}_{2}\left(\mathrm{PMe}_{3}\right)_{2}$-Catalyzed Borylation of Aryl Chlorides. Org. Lett. 2011, 13, 5766-5769. [CrossRef] [PubMed]

23. Molander, G.A.; Cavalcanti, L.N.; García-García, C. Nickel-Catalyzed Borylation of Halides and Pseudohalides with Tetrahydroxydiboron $\left[\mathrm{B}_{2}(\mathrm{OH})_{4}\right]$. J. Org. Chem. 2013, 78, 6427-6439. [CrossRef] [PubMed]

24. Wood, J.L.; Marciasini, L.D.; Vaultier, M.; Pucheault, M. A Synergy for Refunctionalization of Boron. Synlett 2014, 25, 0551-0555. [CrossRef]

25. Xu, L.; Li, P. Direct Introduction of a Naphthalene-1,8-Diamino Boryl [B(dan)] Group by a Pd-Catalysed Selective Boryl Transfer Reaction. Chem. Commun. 2015, 51, 5656-5659. [CrossRef] [PubMed]

26. Yoshida, H.; Takemoto, Y.; Kamio, S.; Osaka, I.; Takaki, K. Copper-Catalyzed Direct Borylation of Alkyl, Alkenyl and aryl Halides with B(dan). Org. Chem. Front. 2017, 4, 1215-1219. [CrossRef]

27. Yoshida, H.; Kamio, S.; Osaka, I. Copper-Catalyzed Borylation of Bromoaryl Triflates with Diborons: Chemoselective Replacement of an Ar-Br Bond. Chem. Lett. 2018, 47, 957-959. [CrossRef]

28. Dang, L.; Lin, Z.; Marder, T.B. Boryl Ligands and Their Roles in Metal-Catalysed Borylation Reactions. Chem. Commun. 2009, 3987-3995. [CrossRef]

29. Hodgson, H.H. The Sandmeyer Reaction. Chem. Rev. 1947, 40, 251-277. [CrossRef]

30. Galli, C. Radical Reactions of Arenediazonium Ions: An Easy Entry into the Chemistry of the Aryl Radical. Chem. Rev. 1988, 88, 765-792. [CrossRef]

31. Mo, F.; Qiu, D.; Zhang, Y.; Wang, J. Renaissance of Sandmeyer-Type Reactions: Conversion of Aromatic C-N Bonds into C-X Bonds ( $\mathrm{X}=\mathrm{B}, \mathrm{Sn}, \mathrm{P}$, or $\left.\mathrm{CF}_{3}\right)$. Acc. Chem. Res. 2018, 51, 496-506. [CrossRef]

32. Mo, F.; Dong, G.; Zhang, Y.; Wang, J. Recent Applications of Arene Diazonium Salts in Organic Synthesis. Org. Biomol. Chem. 2013, 11, 1582-1593. [CrossRef] [PubMed]

33. Mo, F.; Jiang, Y.; Qiu, D.; Zhang, Y.; Wang, J. Direct Conversion of Arylamines to Pinacol Boronates: A Metal-Free Borylation Process. Angew. Chem. Int. Ed. 2010, 49, 1846-1849. [CrossRef] [PubMed]

34. Qiu, D.; Jin, L.; Zheng, Z.; Meng, H.; Mo, F.; Wang, X.; Zhang, Y.; Wang, J. Synthesis of Pinacol Arylboronates from Aromatic Amines: A Metal-Free Transformation. J. Org. Chem. 2013, 78, 1923-1933. [CrossRef] [PubMed]

35. Qiu, D.; Wang, S.; Tang, S.; Meng, H.; Jin, L.; Mo, F.; Zhang, Y.; Wang, J. Synthesis of Trimethylstannyl Arylboronate Compounds by Sandmeyer-Type Transformations and Their Applications in Chemoselective Cross-Coupling Reactions. J. Org. Chem. 2014, 79, 1979-1988. [CrossRef]

36. Qiu, D.; Zhang, Y.; Wang, J. Direct Synthesis of Arylboronic Pinacol Esters from Arylamines. Org. Chem. Front. 2014, 1, 422-425. [CrossRef] 
37. Erb, W.; Albini, M.; Rouden, J.; Blanchet, J. Sequential One-Pot Access to Molecular Diversity through Aniline Aqueous Borylation. J. Org. Chem. 2014, 79, 10568-10580. [CrossRef]

38. Qi, X.; Li, H.-P.; Peng, J.-B.; Wu, X.-F. Borylation of Aryldiazonium Salts at Room Temperature in an Aqueous Solution under Catalyst-Free Conditions. Tetrahedron Lett. 2017, 58, 3851-3853. [CrossRef]

39. Xu, Y.; Yang, X.; Fang, H. Additive- and Photocatalyst-Free Borylation of Arylazo Sulfones under Visible Light. J. Org. Chem. 2018, 83, 12831-12837. [CrossRef]

40. Cid, J.; Carbó, J.J.; Fernández, E. A Clear-Cut Example of Selective Bpin-Bdan Activation and Precise Bdan Transfer on Boron Conjugate Addition. Chem. Eur. J. 2014, 20, 3616-3620. [CrossRef] [PubMed]

41. Yoshida, H.; Takemoto, Y.; Takaki, K. A Masked Diboron in Cu-Catalysed Borylation Reaction: Highly Regioselective Formal Hydroboration of Alkynes for Synthesis of Branched Alkenylborons. Chem. Commun. 2014, 50, 8299-8302. [CrossRef] [PubMed]

42. Yoshida, H.; Takemoto, Y.; Takaki, K. Direct Synthesis of Boron-Protected Alkenyl- and Alkylborons via Copper-Catalyzed Formal Hydroboration of Alkynes and Alkenes. Asian J. Org. Chem. 2014, 3, 1204-1209. [CrossRef]

43. Miralles, N.; Cid, J.; Cuenca, A.B.; Carbo, J.J.; Fernandez, E. Mixed Diboration of Alkenes in a Metal-Free Context. Chem. Commun. 2015, 51, 1693-1696. [CrossRef] [PubMed]

44. Yoshida, H.; Takemoto, Y.; Takaki, K. Borylstannylation of Alkynes with Inverse Regioselectivity: Copper-Catalyzed Three-Component Coupling using a Masked Diboron. Chem. Commun. 2015, 51, 6297-6300. [CrossRef] [PubMed]

45. Kageyuki, I.; Osaka, I.; Takaki, K.; Yoshida, H. Copper-Catalyzed B(dan)-Installing Carboboration of Alkenes. Org. Lett. 2017, 19, 830-833. [CrossRef] [PubMed]

46. Verma, A.; Snead, R.F.; Dai, Y.; Slebodnick, C.; Yang, Y.; Yu, H.; Yao, F.; Santos, W.L. Substrate-Assisted, Transition-Metal-Free Diboration of Alkynamides with Mixed Diboron: Regio- and Stereoselective Access to trans-1,2-Vinyldiboronates. Angew. Chem. Int. Ed. 2017, 56, 5111-5115. [CrossRef] [PubMed]

47. Yan, G.; Huang, D.; Wu, X. Recent Advances in C-B Bond Formation through a Free Radical Pathway. Adv. Synth. Catal. 2018, 360, 1040-1053. [CrossRef]

48. Chen, K.; Wang, L.; Meng, G.; Li, P. Recent Advances in Transition-Metal-Free Aryl C-B Bond Formation. Synthesis 2017, 49, 4719-4730. [CrossRef]

49. Chen, K.; Cheung, M.S.; Lin, Z.; Li, P. Metal-Free Borylation of Electron-Rich Aryl (Pseudo)Halides under Continuous-Flow Photolytic Conditions. Org. Chem. Front. 2016, 3, 875-879. [CrossRef]

50. Chen, K.; Zhang, S.; He, P.; Li, P. Efficient Metal-Free Photochemical Borylation of Aryl Halides under Batch and Continuous-Flow Conditions. Chem. Sci. 2016, 7, 3676-3680. [CrossRef]

51. Jiang, M.; Yang, H.; Fu, H. Visible-Light Photoredox Borylation of Aryl Halides and Subsequent Aerobic Oxidative Hydroxylation. Org. Lett. 2016, 18, 5248-5251. [CrossRef]

52. Liu, W.; Yang, X.; Gao, Y.; Li, C.-J. Simple and Efficient Generation of Aryl Radicals from Aryl Triflates: Synthesis of Aryl Boronates and Aryl Iodides at Room Temperature. J. Am. Chem. Soc. 2017, 139, 8621-8627. [CrossRef] [PubMed]

53. Zhang, L.; Jiao, L. Pyridine-Catalyzed Radical Borylation of Aryl Halides. J. Am. Chem. Soc. 2017, 139, 607-610. [CrossRef] [PubMed]

54. Zhu, C.; Yamane, M. Transition-Metal-Free Borylation of Aryltriazene Mediated by BF 3 . OEt 2 . Org. Lett. 2012, 14, 4560-4563. [CrossRef] [PubMed]

55. Yu, J.; Zhang, L.; Yan, G.-B. Metal-Free, Visible Light-Induced Borykation of Aryldiazonium Salts: A Simple and Green Synthetic Route to Arylboronates. Adv. Synth. Catal. 2012, 354, 2625-2628. [CrossRef]

56. Zhang, J.-M.; Wu, H.-H.; Zhang, J.-L. Cesium Carbonate Mediated Borylation of Aryl Iodides with Diboron in Methanol. Eur. J. Org. Chem. 2013, 6263-6266. [CrossRef]

57. Erb, W.; Hellal, A.; Albini, M.; Rouden, J.; Blanchet, J. An Easy Route to (Hetero)arylboronic Acids. Chem. Eur. J. 2014, 20, 6608-6612. [CrossRef] [PubMed]

58. Zhao, C.-J.; Xue, D.; Jia, Z.-H.; Wang, C.; Xiao, J.-L. Methanol-Promoted Borylation of Arylamines: A Simple and Green Synthetic Method to Arylboronic Acid and Arylboronates. Synlett 2014, 25, 1577-1584. [CrossRef]

59. Ahammed, S.; Nandi, S.; Kundu, D.; Ranu, B.-C. One-pot Suzuki coupling of aromatic amines via visible light photocatalyzed metal free borylation using t-BuONO at room temperature. Tetrahedron Lett. 2016, 57, 1551-1557. [CrossRef] 
60. Pinet, S.; Liautard, V.; Debiais, M.; Pucheault, M. Radical Metal-Free Borylation of Aryl Iodides. Synthesis 2017, 49, 4759-4768. [CrossRef]

61. Mfuh, A.-M.; Nuyen, V.-T.; Chhetri, B.; Burch, J.-E.; Doyle, J.-D.; Nesterov, V.-N.; Arman, H.-D.; Larionov, O.-V. Additive- and Metal-Free, Predictably 1,2- and 1,3-Regioselective, Photoinduced Dual C-H/C-X-Borylation of Haloarenes. J. Am. Chem. Soc. 2016, 138, 8408-8411. [CrossRef]

62. Mfuh, A.-M.; Doyle, J.-D.; Chhetri, B.; Arman, H.-D.; Larionov, O.-V. Scalable, Metal- and Additive-Free, Photoinduced Borylation of Haloarenes and Quaternary Arylammonium Salts. J. Am. Chem. Soc. 2016, 138, 2985-2988. [CrossRef] [PubMed]

63. Iwadate, N.; Suginome, M. Synthesis of masked haloareneboronic acids via iridium-catalyzed aromatic C-H borylation with 1,8-naphthalenediaminatoborane (danBH). J. Orgomet. Chem. 2009, 694, 1713-1717. [CrossRef]

Sample Availability: Samples of the compounds (1a-1t, 2, $\mathbf{3}$ and 4) are available from the authors.

(C) 2019 by the authors. Licensee MDPI, Basel, Switzerland. This article is an open access article distributed under the terms and conditions of the Creative Commons Attribution (CC BY) license (http://creativecommons.org/licenses/by/4.0/). 